\section{VACCINE REGULATION}

\section{Covid-19 vaccines: In the rush for regulatory approval, do we need more data?}

\begin{abstract}
After rollout under emergency authorisation, manufacturers of covid-19 vaccines now have their sights on regulatory approval. But what's the rush, asks Peter Doshi, and is just six months of data from now unblinded trials acceptable?
\end{abstract}

\section{Peter Doshi senior editor}

In April 2021, Pfizer and Moderna announced efficacy results at the six month mark from the phase III trials of their respective covid-19 vaccines. ${ }^{12}$

Pfizer CEO Albert Bourla said the company's data "confirm the favourable efficacy and safety profile of our vaccine and position us to submit a Biologics License Application to the US FDA [Food and Drug Administration]." ${ }^{1}$ And on 7 May it formally initiated that application which, if successful, will earn the Pfizer-BioNTech product, BNT162 b2, the distinction of becoming the first covid-19 vaccine approved by the FDA.

Because lest we forget, all covid-19 vaccines currently in use in the US are available under emergency access only.

(The situation is similar in Europe, where four covid-19 vaccines have been granted "conditional marketing authorisations," a fast track mechanism that can be used in emergencies. These can be converted into standard "marketing authorisations" pending positive data after authorisation, but this has not yet happened for any covid-19 vaccine being administered.)

As hundreds of millions of people around the world get vaccinated, it may seem like wordsmithing to highlight the fact that none of the covid-19 vaccines in use are actually "approved.” Through an emergency access mechanism known as Emergency Use Authorisation (EUA), the products being rolled out still technically remain "investigational."3 Factsheets distributed to vaccinees are clear: "There is no FDA approved vaccine to prevent covid-19."4

The approval-authorisation distinction is often misunderstood by the media, ${ }^{5}$ even in the scientific press. But it was the focus of much discussion back in September 2020. With large phase III trials by Pfizer and Moderna well under way, and the November US presidential election looming, many worried about political pressure resulting in the rollout of an unsafe or ineffective vaccine. ${ }^{6}$

The FDA had already come under fire, accused of bending to the White House in granting EUAs for two covid-19 treatments, hydroxychloroquine and convalescent plasma. But those fears largely dissipated when the FDA published a guidance document in early October outlining its expectations for the EUA. According to the document, at least half of a trial's participants would need to be followed for at least two months. ${ }^{7}$ This alone made it all but certain no vaccine could cross the line before the election.

The FDA also said it would want a vaccine at least $50 \%$ effective (with a confidence interval reaching no lower than $30 \%$ ) against a primary endpoint of preventing SARS-CoV-2 infection or covid-19 disease of any severity ${ }^{8}$ - parameters it had previously defined as necessary for approval. Even for non-clinical parameters, like manufacturing quality, the FDA characterised its expectations for the EUA as "very similar" to those for approval. ${ }^{3}$

\section{Six months: enough?}

One key difference between EUA and approval (also called "licensure," and which for vaccines is known as a BLA (Biologics License Application)) was the expected length of follow-up of trial participants. Unlike its clear articulation of two months for an EUA, the FDA has not committed to a clear minimum for approval.

Cody Meissner, a professor of paediatrics at Tufts University and member of the FDA's advisory committee, was curious. "Is it possible to predict or estimate when conditions of safety and efficacy might be satisfied for BLA?" Meissner asked at the agency's 10 December meeting which had been convened to consider the FDA's first emergency authorisation for the Pfizer vaccine.

The FDA's Doran Fink responded: “I couldn’t predict, but I will say that we typically ask for at least six months of follow-up in a substantial number of clinical trial participants to constitute a safety database that would support licensure."

An approval based on six months of data would represent one of the fastest for a novel vaccine in FDA history. Among the six "first in disease" vaccines approved by the FDA since 2006, pre-licensure pivotal trials were a median of 23 months in duration, according to a recent analysis. ${ }^{9}$

Six months also seems substantially shorter than previously conceptualised expectations. A World Health Organization expert group on covid-19 vaccines (which included FDA regulators) in August 2020 called for follow-up "until at least month 12, or until an effective vaccine is deployed locally."10 Another group, composed of industry and academic authors, similarly wrote in October 2020: "we 
recommend longer term follow-up of all participants ... for at least a year after randomisation." 11

On paper, the phase III studies by Pfizer, Moderna, and Janssen are all of two years' duration. But the FDA's official position on minimum follow-up before licensure is unclear at best.

In its formal guidance last June, the agency said that for licensure applications, it wanted participants followed for covid-19 outcomes for "as long as feasible, ideally at least one to two years" 12 after the first injection. But the same document states that safety assessments for "serious and other medically attended adverse events" should be studied "for at least six months after completion of all study vaccinations. Longer safety monitoring may be warranted for certain vaccine platforms."

Asked to clarify whether its guidance is asking for follow-up of at least six months or one year, a spokesperson told The BMJ: "We do not have any further information beyond what is in the guidance document."

\section{Unblinded and without a control group-what about safety?}

Duration of protection is not the only question that longer, placebo controlled trials can address. They also address vaccine safety.

"Very often, it's the fact that we have that placebo controlled follow-up over time, that gives us the ability to say that the vaccine didn't cause something at a longer period of time after vaccination," the FDA's Philip Krause explained last December. ${ }^{13}$

Yet there is a gap-currently of unknown size but growing-between any expectation of blinded placebo controlled data, and the reality that within weeks of the vaccines receiving an EUA the unblinding of trials commenced as placebo recipients were offered the chance to get vaccinated.

Steven Goodman, associate dean of clinical and translational research at Stanford University, told the FDA in an invited presentation last December, "Once a vaccine is made widely available and encouraged, maintaining a double blinded control group for more than a nominal period is no longer in the investigator's (or regulator's) control and undue pressure to do so may undermine the entire vaccine testing enterprise."14

Goodman's recommendation was to rapidly convert the trials into crossover studies, enabling those on placebo to get vaccinated (and vice versa), while maintaining the blind. The companies challenged the feasibility, calling it "onerous," and a crossover never occurred. ${ }^{15}$

The BMJ asked Moderna, Pfizer, and Janssen (Johnson and Johnson) what proportion of trial participants were now formally unblinded, and how many originally allocated to placebo have now received a vaccine. Pfizer declined to say, but Moderna announced that "as of April 13, all placebo participants have been offered the Moderna covid-19 vaccine and 98\% of those have received the vaccine." ${ }^{2}$ In other words, the trial is unblinded, and the placebo group no longer exists.

Janssen told The BMJ: "We do not have specific figures on how many of our study participants have received a vaccine at this time.” But the company confirmed it was implementing an amended protocol across all countries to unblind all participants in its two phase III trials, the earlier of which passed the median of two month follow-up mark in January.

How the FDA will weigh the loss of blinding and placebo controlled follow-up is unclear, but just months ago the agency said these trial properties were vital.
"Continuation of placebo controlled follow-up after EUA will be important and may actually be critical to ensure that additional safety and effectiveness data are accrued to support submission of a licensure application as soon as possible following an EUA. ... Once a decision is made to unblind an ongoing placebo controlled trial, that decision cannot be walked back. And that controlled follow-up is lost forever," the FDA said last October. ${ }^{3}$

At its next advisory committee in December 2020, the FDA reiterated the importance of the placebo group: "Placebo controlled follow-up can be very important in showing that whatever happened in the vaccine group also happened in the placebo group. Because that's our best way of knowing." 13

\section{What's the rush?}

The US’s “Operation Warp Speed” delivered on its promise to get a novel vaccine into arms in record time (box). Millions of doses of vaccines are being administered daily across the US, making clear that lack of FDA approval is no barrier to access. So just what benefit is there in seeking, and granting, a BLA?

The $B M J$ asked the manufacturers why they were seeking a BLA. Moderna did not respond and Janssen only confirmed it intended to apply for a BLA "later in 2021." Pfizer likewise did not answer but instead quoted an FDA webpage on medical devices, which stated: "Sponsors of EUA products are encouraged to follow up the EUA with a pre-market submission so that it can remain on the market once the EUA is no longer in effect." ${ }^{16}$ But EUAs have no built-in expiry date-in fact, 14 EUAs for Zika diagnostic tests remain active despite the public health emergency expiring in $2017 .{ }^{17}$

Cody Meissner told The BMJ he saw some distinct advantages of a BLA over EUA. An approved vaccine, for one, would provide "an element of assurance," increasing public trust in the vaccines, particularly for those currently sitting on the fence. It would also pave the way for claims of vaccine injury to be routed through a more established compensation programme, and for adding the vaccine to government funded schemes to reach children in financial need. ${ }^{18}$ Finally, it may affect the potential for vaccine mandates: "It is unlikely these vaccines will be mandated while an EUA is in place. Remember that currently these vaccines are still considered experimental.”

While still under EUA, an increasing number of educational and other institutions have already mandated vaccines, but debates over the legality of these actions has hinged on the distinction between authorisation and approval. ${ }^{19}$

But approving a vaccine in order to legally support mandates or convince people of its safety arguably puts the cart before the horse. Meissner responded that a BLA would not be issued until the FDA is convinced of the short and long term safety of these vaccines.

\section{No new biodistribution studies for covid-19 vaccines}

Officials have consistently emphasised that despite shaving years off traditional timelines for producing vaccines, no compromises in the process were taken. ${ }^{20}$ However one type of study, tracking the distribution of a vaccine once injected in the body, was not conducted using any of the three vaccines currently authorised in the US

Such biodistribution studies are a standard element of drug safety testing but "are usually not required for vaccines," according to European

Medicines Agency policy, ${ }^{21}$ which adds, "However, such studies might be applicable when new delivery systems are employed or when the vaccine contains novel adjuvants or excipients." 
In the case of covid-19 vaccines, regulators accepted biodistribution data from past studies performed with related, mostly unapproved compounds that use the same platform technology. $22-25$

Janssen told The $B M$ J its covid-19 vaccine leverages the same technology as its Ebola vaccine, which received licensure last June. "Our confidence in our adenovirus vector Ad26 is based on our experience with this vector."

Pfizer and Moderna did not respond to The BMJ's questions regarding why no biodistribution studies were conducted on their novel mRNA products, and none of the companies, nor the FDA, would say whether new biodistribution studies will be required prior to licensure.

Competing interests $\mathrm{PD}$ gave a public statement at the October and December FDA advisory committee meetings mentioned in this article (transcripts here: https://faculty.rx.umary-

land.edu/pdoshi/\#publications), and may continue to engage in public input towards regulatory decision making around covid-19 vaccines. PD is also employed by a university that has mandated covid-19 vaccines for all faculty, staff, and students. The views and opinions expressed here are those of the author and do not necessarily reflect official policy or position of the University of Maryland.

1 Pfizer. Pfizer and BioNTech confirm high efficacy and no serious safety concerns through up to six months following second dose in updated topline analysis of landmark COVID-19 vaccine study. 2021. https://investors.pfizer.com/investor-news/press-release-details/2021/Pfizer-andBioNTech-Confirm-High-Efficacy-and-No-Serious-Safety-Concerns-Through-Up-to-Six-MonthsFollowing-Second-Dose-in-Updated-Topline-Analysis-of-Landmark-COVID-19-VaccineStudy/default.aspx

2 Moderna, Inc. Moderna provides clinical and supply updates on covid-19 vaccine program ahead of 2nd annual vaccines day. 2021. https://investors.modernatx.com/news-releases/news-releasedetails/moderna-provides-clinical-and-supply-updates-covid-19-vaccine

3 US Food and Drug Administration. 161st Vaccines and Related Biological Products Advisory Committee (VRBPAC) meeting. 2020. https://www.fda.gov/media/143982/download

4 Moderna TX. Inc. Moderna covid-19 vaccine EUA fact sheet for recipients and caregivers. 2021. https://www.fda.gov/media/144638/download

5 Kaplan K. The FDA didn't "approve” Pfizer's covid-19 vaccine. Here’s why. Los Angeles Times 2020. https://www.latimes.com/science/story/2020-12-12/why-fda-didnt-approve-pfizer-covid19-vaccine-eua

6 Avorn J, Kesselheim A. Regulatory decision-making on covid-19 vaccines during a public health emergency. JAMA 2020;324:1284-5. doi: 10.1001/jama.2020.17101 pmid: 32870268

7 US Food and Drug Administration. Development, authorization and licensure of vaccines to prevent COVID-19. October 6, 2020. https://www.fda.gov/media/142723/download

8 Doshi P. Will covid-19 vaccines save lives? Current trials aren't designed to tell us. BMJ 2020;371:m4037. doi: 10.1136/bmj.m4037 pmid: 33087398

9 Kesselheim AS, Darrow J], Kulldorff M, etal. An overview of vaccine development, approval, and regulation, with implications for covid-19. Health Aff (Millwood) 2021;40:25-32. doi: 10.1377/hlthaff.2020.01620 pmid: 33211535

10 Krause P, Fleming TR, Longini I, Henao-Restrepo AM, Peto R; World Health Organization Solidarity Vaccines Trial Expert Group. COVID-19 vaccine trials should seek worthwhile efficacy. Lancet 2020;396:741-3. doi: 10.1016/S0140-6736(20)31821-3 pmid: 32861315

11 Mehrotra DV, Janes HE, Fleming TR, etal. Clinical endpoints for evaluating efficacy in covid-19 vaccine trials. Ann Intern Med 2021;174:221-8. doi: 10.7326/M20-6169 pmid: 33090877

12 US Food and Drug Administration. Development and licensure of vaccines to prevent covid-19: guidance for industry. 2020. https://www.fda.gov/media/139638/download

13 US Food and Drug Administration. 162nd Vaccines and Related Biological Products Advisory Committee (VRBPAC) meeting. 2020. https://www.fda.gov/media/144859/download

14 Goodman S. Considerations for placebo-controlled trial design if an unlicensed vaccine becomes available. Vaccines and related biological products advisory committee. 2020. https://www.fda.gov/media/144354/download

15 Lenzer J. Covid-19: Should vaccine trials be unblinded?BMJ 2020;371:m4956. doi: 10.1136/bmj.m4956 pmid: 33376088

16 US Food and Drug Administration. FAQs on emergency use authorizations (EUAs) for medical devices during the covid-19 pandemic. 2021. https://www.fda.gov/medical-devices/coronavirusdisease-2019-covid-19-emergency-use-authorizations-medical-devices/faqs-emergency-useauthorizations-euas-medical-devices-during-covid-19-pandemic

17 Zuckerman DM. Emergency use authorizations (EUAs) versus FDA approval: implications for covid-19 and public health. Am J Public Health 2021;111:1065-9. doi: 10.2105/AIPH.2021.306273 pmid: 33950730

18 Meissner HC. A viral pandemic, vaccine safety, and compensation for adverse events. JAMA 2021:325:721-2. doi: 10.1001/jama.2020.26792 pmid: 33544127

19 Musumeci M, Kates J. Key questions about covid-19 vaccine mandates. 2021. https://www.kff.org/coronavirus-covid-19/issue-brief/key-questions-about-covid-19-vaccinemandates/

20 Nania R. FDA commissioner Stephen Hahn on covid vaccine safety. 2020.

https://www.aarp.org/health/drugs-supplements/info-2020/coronavirus-vaccine-safety.html
21 European Medicines Agency. Guideline on clinical evaluation of new vaccines (EMEA/CHMP/NWP/164653/2005) 2006. https://www.ema.europa.eu/en/documents/scientificguideline/guideline-clinical-evaluation-new-vaccines_en.pdf

22 European Medicines Agency. Assessment Report. Comirnaty (COVID-19 mRNA vaccine (nucleoside-modified)), EMA/707383/2020 Corr.1.2021. https://www.ema.europa.eu/en/documents/assessment-report/comirnaty-epar-public-assessment-report_en.pdf\#page=45

23 European Medicines Agency. Assessment Report. COVID-19 Vaccine Moderna (COVID-19 mRNA Vaccine (nucleoside-modified)), EMA/15689/2021 Corr.12021. https://www.ema.europa.eu/en/documents/assessment-report/covid-19-vaccine-moderna-epar-public-assessment-report_en.pdf\#page= 47

24 European Medicines Agency. Assessment Report. COVID-19 Vaccine Janssen, EMA/158424/2021 2021. https://www.ema.europa.eu/en/documents/assessment-report/covid-19-vaccine-janssenepar-public-assessment-report_en.pdf\#page $=50$

25 Pfizer. SARS-CoV- 2 mRNA Vaccine (BNT162, PF-07302048) 2.6.4 Yakubutsu dōtai shiken no gaiyō bun [summary of pharmacokinetic studies]. https://www.pmda.go.jp/drugs/2021/P20210212001/672212000_30300AMX00231_|100_1.pdf\#page=16 